\section{(1)}

CrossMark

\title{
Management of repository corticotrophin injection therapy for pulmonary sarcoidosis: a Delphi study
}

\author{
Franck F. Rahaghi ${ }^{1}$, Nadera J. Sweiss $\mathbb{1}^{2}$, Lesley Ann Saketkoo ${ }^{3}$, \\ Mary Beth Scholand ${ }^{4}$, Joseph B. Barney ${ }^{5}$, Alicia K. Gerke ${ }^{6}$, Elyse E. Lower ${ }^{7}$, \\ Mehdi Mirsaeidi ${ }^{8}$, Lanier O'Hare ${ }^{5}{ }^{5}$, Mark J. Rumbak ${ }^{9}$, Lobelia Samavati ${ }^{10}$ and \\ Robert P. Baughman ${ }^{7}$
}

Affiliations: ${ }^{1}$ Cleveland Clinic Florida, Weston, FL, USA. ${ }^{2}$ University of Illinois at Chicago College of Medicine, Chicago, IL, USA. ${ }^{3}$ Tulane University School of Medicine, New Orleans, LA, USA. ${ }^{4}$ University of Utah Medical Center, Salt Lake City, UT, USA. ${ }^{5}$ University of Alabama at Birmingham, Birmingham, AL, USA. ' University of lowa Carver College of Medicine, lowa City, IA, USA. "University of Cincinnati Medical Center, Cincinnati, OH, USA. ${ }^{8}$ University of Miami Miller School of Medicine, Miami, FL, USA. ${ }^{9}$ University of South Florida College of Medicine, Tampa, FL, USA. ${ }^{10}$ Wayne State University School of Medicine, Detroit, MI, USA.

Correspondence: Robert P. Baughman, University of Cincinnati Medical Center, 1001 Holmes Building, 200 Albert Sabin Way Cincinnati, OH 45267-0565. E-mail: baughmrpđucmail.uc.edu

@ERSpublications

In this paper, a modified Delphi method was used to develop an expert consensus on the use of repository corticotrophin injection therapy for pulmonary sarcoidosis, including dosing, concomitant medications, contraindications and adverse event management. http://bit.ly/2TyauZp

Cite this article as: Rahaghi FF, Sweiss NJ, Saketkoo LA, et al. Management of repository corticotrophin injection therapy for pulmonary sarcoidosis: a Delphi study. Eur Respir Rev 2020; 29: 190147 [https://doi. org/10.1183/16000617.0147-2019].

ABSTRACT In patients treated with repository corticotrophin injection (RCI) for pulmonary sarcoidosis, effective management of adverse events may improve adherence. However, management of adverse events may be challenging due to limitations in real-world clinical experience with RCI and available published guidelines.

We surveyed 12 physicians with a modified Delphi process using three questionnaires. Questionnaire 1 consisted of open-ended questions. Panellists' answers were developed into a series of statements for Questionnaires 2 and 3. In these, physicians rated their agreement with the statements using a Likert scale.

Key consensus recommendations included a starting dose of 40 units twice a week for patients with less severe disease, continued at a maintenance dose for patients who responded, particularly those with chronic refractory sarcoidosis. Panellists reached consensus that concomitant steroids should be quickly tapered in patients receiving RCI, but that concomitant use of immunosuppressive medications should be continued. Panellists developed consensus recommendations for adverse event management, and reached consensus that RCI should be down-titrated or discontinued if other interventions for the adverse effects fail or if the adverse effect is severe.

In the absence of clinical evidence, our Delphi consensus opinions may provide practical guidance to physicians on the management of RCI to treat pulmonary sarcoidosis.

This article has supplementary material available from err.ersjournals.com

Provenance: Publication of this peer-reviewed article was sponsored by Mallinckrodt Pharmaceuticals, UK (principal sponsor, European Respiratory Review issue 155).

Received: 05 Nov 2019 | Accepted after revision: 19 Feb 2020

Copyright $\odot$ ERS 2020. This article is open access and distributed under the terms of the Creative Commons Attribution Non-Commercial Licence 4.0. 


\section{Introduction}

Sarcoidosis is a multisystem granulomatous disorder of unknown cause $[1,2]$. As a systemic heterogeneous inflammatory disease, it is characterised by the presence of non-caseating epithelioid granulomas in one or multiple organs, with the lung affected in almost $90 \%$ of cases [3]. Lung involvement commonly manifests as bilateral hilar lymphadenopathy and pulmonary infiltration, with the development of pulmonary fibrosis in more severe cases. For most patients, pulmonary sarcoidosis may be a benign and self-limiting disorder, and spontaneous remission occurs in the majority of patients with asymptomatic disease [4]. However, for a significant minority of patients, sarcoidosis is a chronic, debilitating and even life-threatening condition. It may affect the skin, eyes, liver and/or lymph nodes [5, 6]. Cardiac and neurologic involvement may also occur and may cause morbidity and death [4]. Mortality rates up to 6\% have been reported, with deaths attributed to respiratory, neurologic or cardiovascular system involvement $[7,8]$. Reduction in mortality and the prevention of disability due to major organ involvement are treatment goals. Thus, it is important to recognise severe disease and identify patients at higher risk of progression to severe disease [9].

Although the optimal treatment for sarcoidosis remains unclear, corticosteroid therapy has been the mainstay of therapy for those with significantly symptomatic or progressive pulmonary disease or serious extrapulmonary disease $[10,11]$. However, there is no evidence that oral corticosteroids improve patient-oriented outcomes including mortality, or that they have any effect on long-term outcomes, lung function, radiologic findings or disease progression [10]. Second- and third-line therapies for pulmonary sarcoidosis are often used as alternatives to steroids and include antimetabolites (methotrexate, azathioprine, leflunomide and mycophenolate), followed by biologic agents such as tumour necrosis factor (TNF)- $\alpha$ inhibitors, which can control disease and may be particularly useful in managing advanced disease $[10,12,13]$. Antimetabolites and biologic agents are typically reserved for patients with corticosteroid refractory disease, intolerable adverse effects or toxicity from corticosteroids, and for patients who choose not to take corticosteroids [10].

This article focuses on the use of Acthar Gel (repository corticotrophin injection (RCI)) in the management of pulmonary sarcoidosis. Acthar Gel is a naturally sourced complex mixture of adrenocorticotropic hormone analogs and other pituitary peptides. The Acthar Gel manufacturing process converts the initial porcine pituitary extract with low ACTH content into a mixture having modified porcine ACTH and other related peptide analogs solubilised in gelatin. A major component in the formulated complex mixture is $\mathrm{N}-25$ deamidated porcine ACTH (1-39). RCI has been used to treat autoimmune and inflammatory conditions since the 1950s [14, 15]. To date, RCI has received approval from the US Food and Drug Administration for the treatment of 19 conditions, including symptomatic pulmonary sarcoidosis. A synthetic analogue known as tetracosactide has received regulatory approval for the treatment of Duchenne muscular dystrophy in parts of the European Union. RCI was previously approved for the treatment of sarcoidosis in Europe, but to the authors' knowledge it is not marketed in Europe or other regions outside the USA at present. However, a synthetic corticotropin is currently being studied in England and Europe. Given the increasing use of RCI in the USA and a double-blind placebo-controlled trial currently underway in the USA, the drug has become increasingly used and discussed in patient management and is likely to become a treatment option in Europe in the near future.

There have been few contemporary reports of the use of RCI for sarcoidosis $[16,17]$, and only minimal information is available on the dosage and toxicity of RCI in treating patients with chronic sarcoidosis. The product labelling includes little guidance on dosing or the management of common and important adverse effects, leaving physicians who manage sarcoidosis with relatively little direction on the practical use of RCI. In addition, physicians may need guidance on maintenance therapy, concomitant steroid therapy, dose reduction or discontinuation and other practical aspects of the use of RCI. In the absence of clinical evidence on these topics, expert opinion may be a useful guide for clinical management for physicians who treat sarcoidosis patients with RCI. This study is intended to provide consensus recommendations for the management of sarcoidosis with RCI based on the current practice in the USA through the use of a modified Delphi process. Because RCI is not widely used outside the USA, the Delphi panel was intentionally limited to US-based physicians who are expert in sarcoidosis and have significant experience with RCI in their practice, excluding physicians in the USA and Europe who have not prescribed RCI for sarcoidosis.

\section{Methods}

We performed a modified Delphi study to determine if there was expert consensus regarding use of RCI in terms of dosage and adverse event management in pulmonary sarcoidosis. This process allows development of consensus recommendations even if the scientific body of literature is sparse and the published experience with a treatment modality is limited or non-existent [18]. In our modified Delphi 


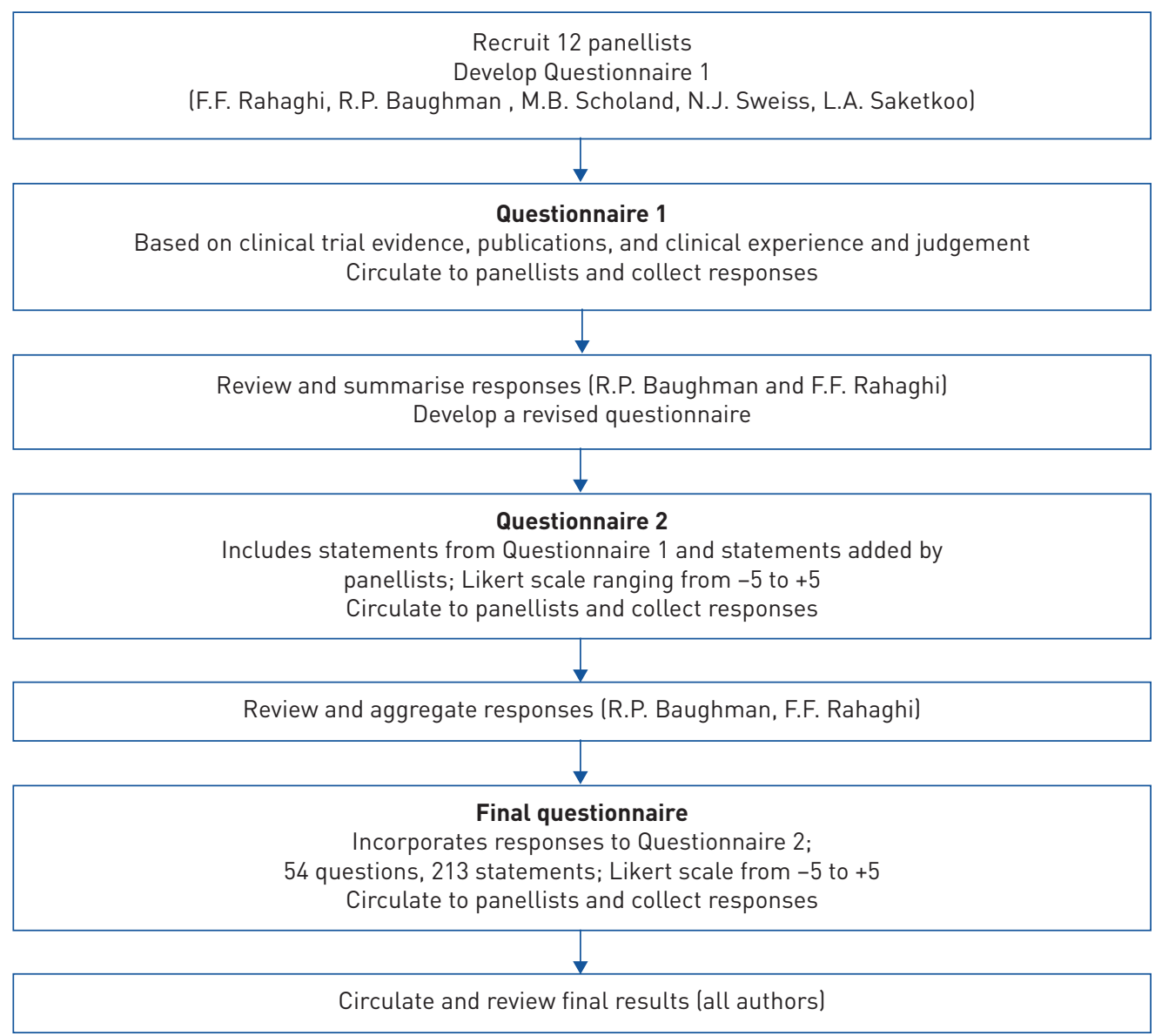

FIGURE 1 The Delphi process used in the study.

approach, a group of expert clinicians and other appropriate stakeholders collaborated in a coordinated multistep process focused on the completion of three questionnaires (figure 1). The study was initiated by the lead and corresponding authors (F.F. Rahaghi and R.P. Baughman), who worked with a panel of other expert pulmonologists (L.A. Saketkoo, M.B. Scholand and N.J. Sweiss) to develop the initial questionnaire, determine criteria for panellist selection, and identify potential panellists. Potential panellists were screened and recruited as physicians who treat patients with pulmonary sarcoidosis. The actual number of patients with sarcoidosis under active management and the number of patients treated with RCI were verified by panellist self-report. All panellists who participated actively in the Delphi process (defined as completing and returning a minimum of two out of three questionnaires including the final (third) questionnaire and reviewing the draft and final manuscripts) met the International Committee of Medical Journal Editors criteria for authorship and are included as co-authors.

The first questionnaire was developed by F.F. Rahaghi and R.P. Baughman based on the RCI package insert, current clinical practice evidence, review of the literature and clinical experience. The resulting questions were reviewed by L.A. Saketkoo, M.B. Scholand and N.J. Sweiss and finalised as a topically organised questionnaire at a roundtable meeting. The first questionnaire was distributed to all 12 panellists by email. Panellists were requested to independently provide responses to each question and/or statement and to add additional relevant comments and/or statements at their discretion. Panellists were also encouraged to comment on the validity, specificity and content of the items under consideration. Panellists' answers were analysed and developed into potential consensus statements.

The moderators (F.F. Rahaghi and R.P. Baughman) reviewed and summarised responses to the first questionnaire and used the responses to develop a second questionnaire that incorporated the initial statements and additional statements added by the panellists without changing the overall organisation. Panellists were requested to rate each statement on a numerical Likert scale ranging from -5 (strongly disagree) to +5 (strongly agree). Panellists were also requested to provide comments on each statement and to add additional relevant statements at their discretion. The results of the second questionnaire identified areas of preliminary consensus. 
The third and final questionnaire was similar to the second questionnaire, and included the panellists' individual responses and aggregated results of the second questionnaire (mean and standard deviation of Likert scale scores). Providing this information is intended to promote consensus by making participants aware of the group's opinion, in accordance with the purpose of the questionnaire, which was to build consensus "for" or "against" in statements wherein consensus can potentially be reached.

Following standard practice for the Delphi methodology, panellists' anonymity was maintained throughout the process. Anonymity is deemed essential in Delphi methodology in order to prevent bias by influential clinicians and to reduce the pressure towards conformity.

Consensus was defined prospectively as a mean rating of $\geqslant 2.5$ or $\leqslant-2.5$ on the Likert scale with a standard deviation that did not cross zero (supplementary appendix 1).

All comments were incorporated anonymously in the statements for Likert scale evaluation and questionnaires distributed to participants in each round. All literature referenced in the statements was circulated to the entire panel for reference and independent examination.

\section{Results}

The Delphi panel consisted of 12 physicians, all of whom participated in the Delphi process and had prescribed RCI in their clinical practice. The panellists' characteristics are shown in table 1. All panellists listed sarcoidosis as a primary clinical and research interest, have been treating sarcoidosis for 5-35 years, and have treated at least several hundred patients with sarcoidosis. Panellists initiated RCI therapy for a variety of reasons, including intolerance to other agents and as second-, third- or fourth-line therapy (figure 2). The final questionnaire contained 213 statements that panellists evaluated on a Likert scale, and six questions intended to gather information. The Delphi panel reached consensus on 104 (48.8\%) out of the 213 statements. The full questionnaire and aggregated results are presented in supplementary appendix 2.

Dosing

The panel's consensus recommendations for dosing and concomitant steroid use are summarised in figure 3. The panel did not reach consensus on the need for an RCI loading dose (mean \pm SD-1.5 \pm 2.8 ). The starting dose of 40 units twice weekly for most patients achieved consensus (3.0 22.1 ) for those with less severe disease, but panellists failed to achieve consensus whether a higher initial dose was indicated with a more active/severe condition (1.08 \pm 3.09$)$. RCI should be continued at a maintenance dose for most

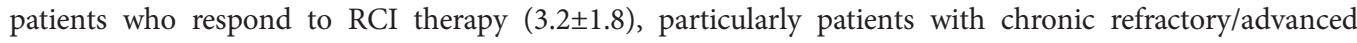
sarcoidosis $(3.5 \pm 1.7)$. There was a consensus that the maintenance dose should be individualised for each patient (3.2 \pm 1.5$)$.

\section{TABLE 1 Characteristics of the Delphi panellists}

Characteristic

Panellists n

$\begin{array}{ll}\text { Completed survey } & \\ \text { Survey } 1 & 12 \\ \text { Survey } 2 & 11 \\ \text { Survey } 3 & 12 \\ \text { Specialty } & 7 \\ \quad \text { Pulmonology } & 4 \\ \text { Internal medicine } & 1 \\ \text { Primary care/family medicine } & 2 \\ \text { Experience treating sarcoidosis } & 4 \\ \quad 6-10 \text { years } & 2 \\ 11-20 \text { years } & 3 \\ >20 \text { years } & 2 \\ \text { Patients with sarcoidosis treated in entire career } \mathbf{n} & 3 \\ <500 & \\ 501-1000 & 1 \\ >1000 & 3 \\ \text { Patients with sarcoidosis treated last year } \mathbf{n} & 4 \\ <50 & \\ 51-150 & \\ >150 & \end{array}$

\footnotetext{
${ }^{\#}$ : some panellists reported multiple specialties.
} 


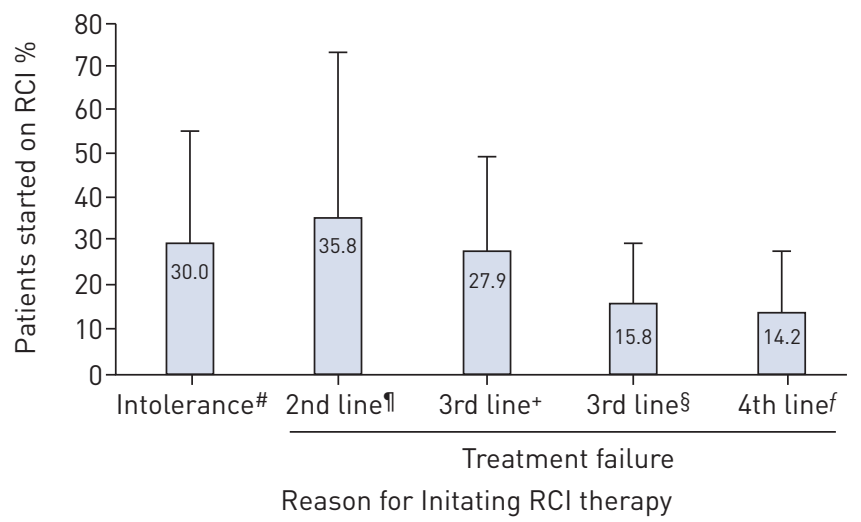

FIGURE 2 Panellists' reasons for initiating repository corticotrophin injection (RCI) in patients with pulmonary sarcoidosis. \#: to steroids, antimetabolites or biologics; " : after steroids; ${ }^{+}$: after steroids and antimetabolites: $\S$ : after steroids and biologics; ${ }^{f}$ : after steroids, antimetabolites and biologics.

The panel recommended reducing or discontinuing RCI if it: is ineffective in achieving the goals of

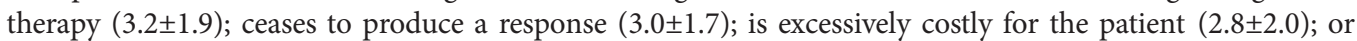
if the patient develops major steroid adverse effects (3.4 \pm 1.6$)$, hyperglycaemia or other diabetes

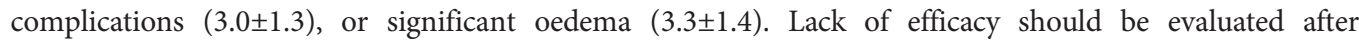
3-6 months of RCI therapy (4.3 \pm 1.4$)$. Weaning to the lowest efficacious RCI dose should be considered if the patient has stable, well-controlled disease after 6-12 months of therapy (3.1 \pm 1.7$)$, and should be performed by decreasing the dosing frequency, and then the dose (2.6 \pm 1.9$)$.

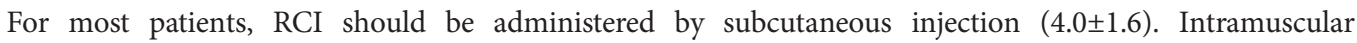
injection may be used if subcutaneous injection fails $(3.1 \pm 1.8)$. The following tests should be done (or recent results should be available) before initiation of RCI: complete blood count (2.7 \pm 2.3$)$, comprehensive metabolic panel (3.3 \pm 2.4$)$ and bone density scan (dual-energy $\mathrm{x}$-ray absorptiometry) $(2.8 \pm 1.8)$.

\section{Concomitant medications}

Panellists reached consensus that concomitant steroids should be tapered and potentially weaned in patients

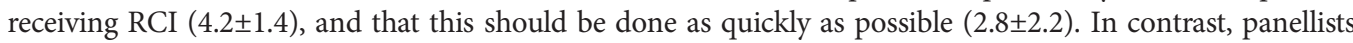
recommended continuing concomitant use of immunosuppressive medications (methotrexate, azathioprine

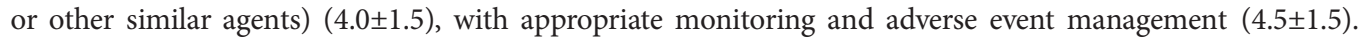
Associated recommendations included: maintain steady RCI until a concomitant immunosuppressant is on board (3.1 \pm 1.5$)$, decrease immunosuppressant dose if possible depending on which medications are used (2.6 \pm 1.8$)$, keep disease-modifying anti-rheumatic drugs unchanged while patient is taking RCI (3.2 \pm 1.6$)$,

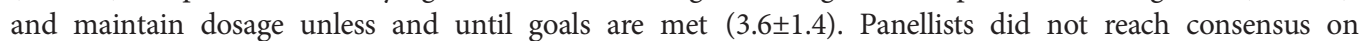
whether the dose of concomitant medications should be reduced when RCI is initiated (0.1 \pm 2.6$)$, although the level of consensus varied for several related questions (supplementary appendix 3).

\section{Contraindications}

The Delphi panel reached consensus that RCI is contraindicated in patients with severe or uncontrolled

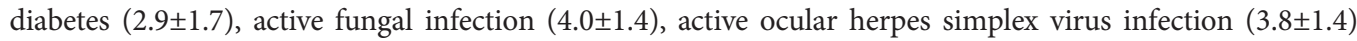

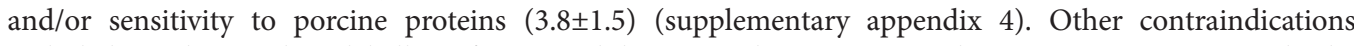
included in the product labelling for RCI did not reach consensus. This is commensurate with the knowledge that most of these adverse events listed on the package insert are extrapolated from the expected steroid-like adverse events and not derived from a controlled trial of RCI.

\section{Hospitalised patients}

Panellists reached consensus that RCI should be continued during hospitalisation in the absence of adverse events potentially related to RCI (3.3 \pm 2.9$)$, life-threatening infection (3.4 \pm 3.0$)$ and/or sepsis $(3.2 \pm 2.9)$. Patients should receive steroids at a stress dose during hospitalisation (3.1 \pm 1.9$)$ (supplementary appendix 5). The authors also recommend stress dose steroids if haemodynamic insufficiency is present, although this point was not specifically addressed in the Delphi process.

\section{Adverse event management}

The panel considered several classes of adverse events including oedema, anxiety/depression, infection, increased appetite or weight gain, glucose intolerance or worsening in glycaemic control, hypertension, 
Mean \pm SD

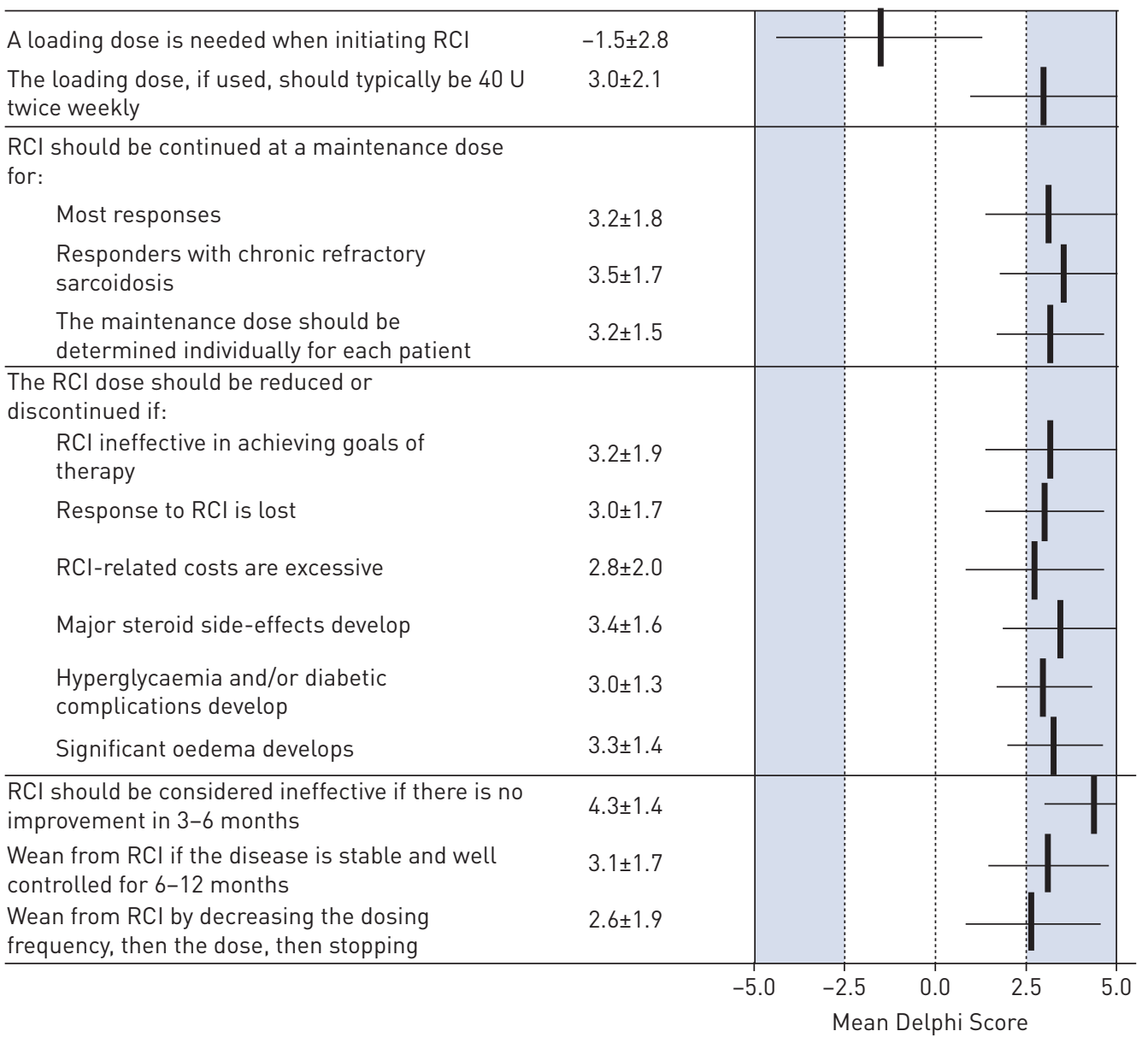

FIGURE 3 Consensus recommendations for repository corticotrophin injection (RCI) dosing and concomitant steroid use.

darkening of the skin, other skin-related adverse events, localised injection site pain and insomnia. Strategies for managing adverse events were categorised as non-pharmacologic, pharmacologic, and RCI dose modification or discontinuation.

For most of these adverse events, the Delphi panel reached consensus that RCI should be down-titrated or discontinued if the other recommended interventions fail or if the adverse event is severe and significant. Panellists did not reach consensus on resuming or up-titrating RCI after resolution for any adverse event. Exceptions are mentioned hereafter, and details on the strength of consensus are provided in supplementary appendix 2 .

Consensus recommendations for management of oedema are summarised in figure 4 . The cause of the oedema should be evaluated and diagnosed (4.4 \pm 1.4$)$. Patients should be instructed to limit salt intake $(4.0 \pm 1.5)$ and elevate the affected $\operatorname{limb}(s)(3.7 \pm 1.4)$, and diuretics should be initiated (3.6 \pm 1.6$)$, possibly loop diuretics $(2.9 \pm 1.9)$ or spironolactone $(2.8 \pm 2.0)$.

Consensus recommendations for management of anxiety and/or depression are also summarised in figure 4 . The patient should be referred to their primary care physician or psychiatrist for management of these conditions $(2.7 \pm 2.6)$. The panellists considered but did not reach consensus on use of anxiolytic therapy (1.0 \pm 1.6$)$, antidepressants $(1.8 \pm 1.8)$ or antipsychotics $(-0.7 \pm 2.3)$.

For infection, the panel reached consensus that pharmacologic therapy should be used as first-line

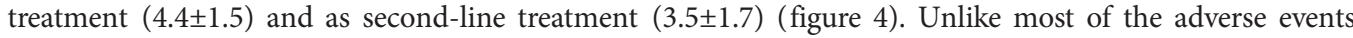
considered, panellists did not reach consensus on down-titrating the RCI dose after other interventions fail $(2.4 \pm 3.0)$, suggesting that immediate down-titration may be appropriate.

Consensus recommendations for increased appetite or weight gain included behavioural intervention

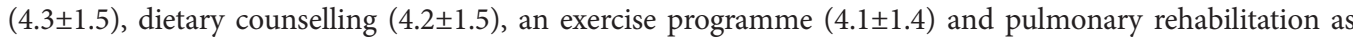




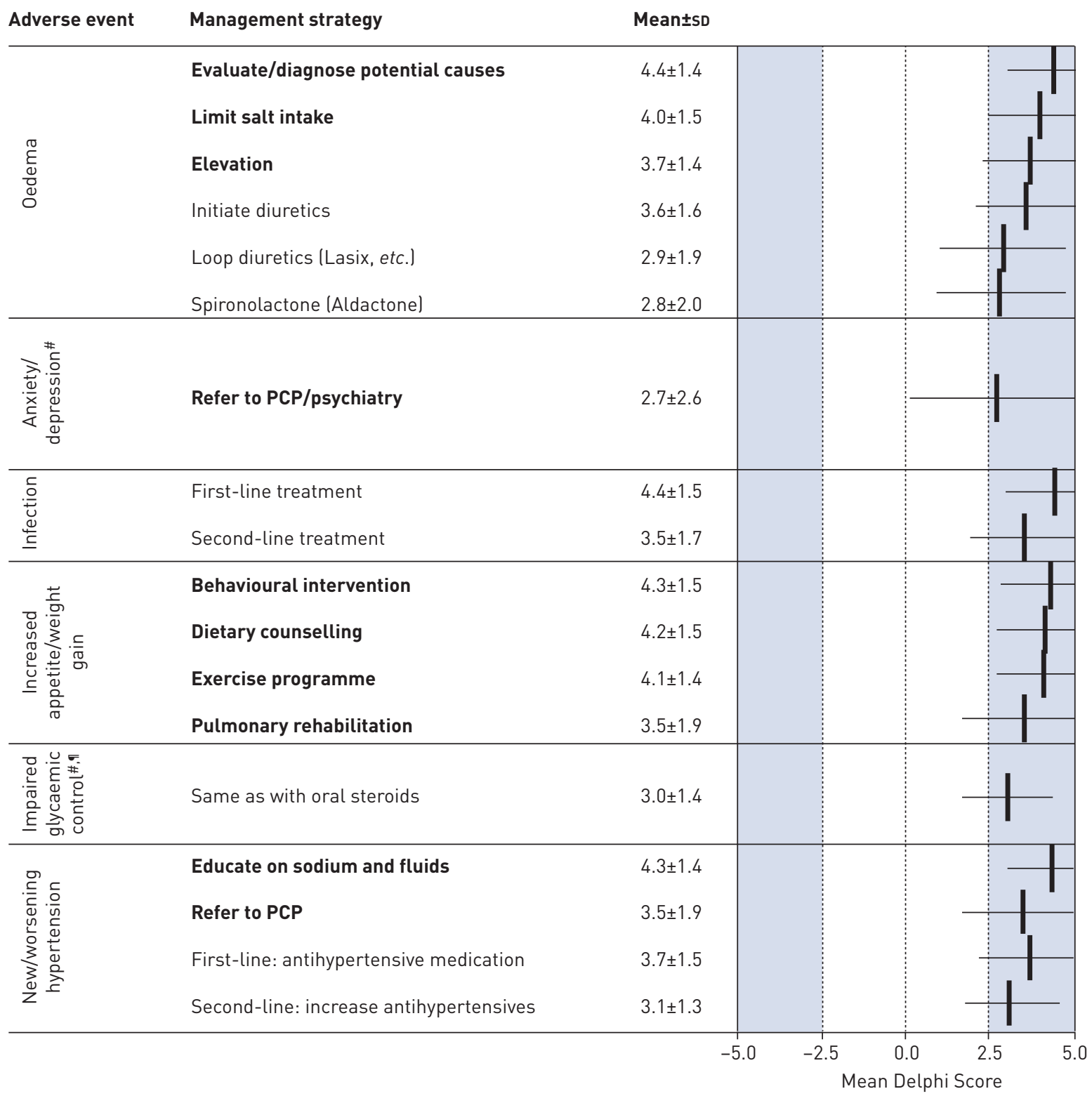

FIGURE 4 Consensus recommendations for non-pharmacological and pharmacological management of new or worsening adverse events in patients receiving repository corticotrophin injection as therapy for pulmonary sarcoidosis. Bold indicates non-pharmacological interventions. PCP: primary care physician. " : no pharmacological interventions reached consensus for these adverse events; " glucose intolerance or worsening of glucose control.

a specific exercise programme where appropriate (3.5 \pm 1.9$)$ (figure 4). Pharmacologic intervention with appetite suppressants was considered but did not reach consensus.

Panellists reached consensus that glucose intolerance or worsening in glycaemic control should be

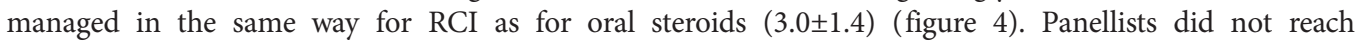
consensus on the use of specific medications.

Patients who develop hypertension should be educated regarding sodium and fluids $(4.3 \pm 1.4)$ and referred to their primary care physician (3.5 \pm 1.9$)$. Panellists recommended use of first-line antihypertensive therapies (3.7 \pm 1.5$)$ and second-line antihypertensive therapies if needed (3.1 \pm 1.3$)$ (figure 4).

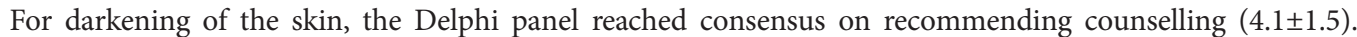
Unlike most of the other adverse events considered, panellists barely reached consensus on recommending discontinuation if other interventions failed (2.5 \pm 1.7$)$. For other skin-related adverse events, the panel recommended counselling and encouraging the patient to complete RCI therapy $(3.3 \pm 2.3)$, rotation of the

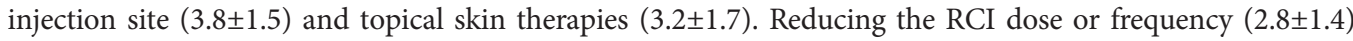


and referral to dermatology (3.1 \pm 1.8$)$ were recommended as second- and third-line strategies. Panellists did not reach consensus for discontinuation of RCI if other interventions failed (1.8 \pm 2.1$)$.

Panellists reached consensus that localised injection site pain can be managed by cooling the skin with an

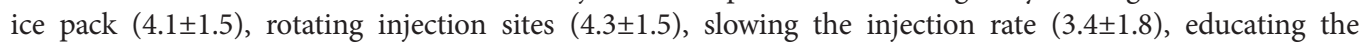
patient on injection technique $(4.3 \pm 1.4)$ and massaging the area $(2.8 \pm 2.1)$. With regard to RCI dose modifications, panellists reached consensus that RCI should be down-titrated or discontinued for severe and significant adverse events. Unlike the other adverse events considered, they did not reach consensus on down-titrating or discontinuing RCI if other interventions failed.

Consensus recommendations for insomnia included sleep hygiene $(4.14 \pm 1.4)$, mindfulness techniques and

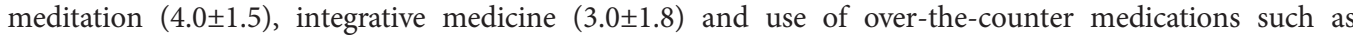
antihistamines and melatonin (3.5 \pm 1.5$)$. Panellists did not reach consensus on down-titration if other interventions failed $(2.0 \pm 1.5)$.

\section{Discussion}

The Delphi process yielded consensus opinions on the use of RCI in treating pulmonary sarcoidosis based on the combined clinical experience of experts in the field. The Delphi process is often used to develop and record consensus advice in situations where evidence is not available [19-21]. For this study, the lead author (F.F. Rahaghi) and senior author (R.P. Baughman) have extensive experience organising initial panels of experts and have previous experience conducting Delphi studies that have resulted in publications that are used by physicians in clinical practice. The prior studies produced consensus recommendations for the use of oral treprostinil for pulmonary arterial hypertension [22] and recommendations on the clinical management of pulmonary arterial hypertension patients receiving macitentan [23], in addition to the recommendations on a treatment algorithm for sarcoidosis in this series [13].

The Delphi panel was able to reach consensus on slightly less than half of the statements discussed. This lack of consensus reflects the lack of clinical evidence on key points related to the use of RCI in sarcoidosis, and highlights areas where original research is needed to help guide evidence-based practice. In areas where the we did reach consensus, we believe our suggestions on contextualising adverse effect and their management, dose escalation and de-escalation, and many other practical points may be useful in helping less experienced clinicians better use the medication in the appropriate patient.

There have been three relatively large series reporting on the effectiveness of RCI in treating sarcoidosis. The retrospective study by Baughman et al. studied 47 patients, of whom 29 received at least three months of therapy. Of those 29 , more than ninety percent had either improvement or significant steroid reduction. RCI was found to be steroid sparing in advanced sarcoidosis. However, significant toxicity was encountered in over one-third of the patients [16]. In a prospective study of RCI for pulmonary sarcoidosis, BAUGHMAN et al. [17] reported on 16 patients who completed 24 weeks of RCI therapy. There was a significant reduction in prednisone dose, associated with significant improvement in patient-reported quality of life. The study also found that an RCI dosage of 40 units twice a week was as effective as 80 units and appeared to be better tolerated [17]. In another retrospective study, CHOPRA et al. [24] reviewed 302 sarcoidosis patients and reported that $>90 \%$ had improvement of their symptoms. Note that this study had not yet been published when the Delphi panels were conducted.

Although RCI has been approved to treat inflammatory and autoimmune diseases for more than 60 years, recent progress in understanding melanocortin receptors and the effects of RCI in modulating immune responses has led to increased interest in RCI as a therapeutic choice [25]. The role of RCI in the overall treatment algorithm is considered in the article on the treatment algorithm for sarcoidosis in this issue of the European Respiratory Review [26]. RCI was considered a "fourth-line" therapy for patients who were not able to take "third-line" treatments such as infliximab or other anti-TNF therapies. The anti-TNF therapies have been associated with significant toxicity and occasional failure. One report found that half of sarcoidosis patients started on infliximab eventually had the drug discontinued [27]. In addition, there are contraindications to the use of anti-TNF therapies, such as malignancy or demyelinating disease. In those cases, RCI was considered an acceptable option.

Our Delphi panel did not achieve consensus on use of a loading dose, but recommended the 40 unit dosage for the less severe conditions, with suggestion of individualising dose for the severity of illness.

Steroids should be weaned as rapidly as possible after the start of RCI treatment. Dose reduction should be considered for excessive adverse effects. The maintenance dosage should be continued for at least 612 months before down-titration is attempted. RCI may be considered ineffective if no positive effect is observed within 3-6 months. With years of experience, the RCI product label has accumulated many contraindications to treatment for various indications, but not all contraindications may be associated with 
all indications. The panellists considered active fungal infections, ocular herpes and uncontrolled diabetes to be contraindications to RCI use for treatment of pulmonary sarcoidosis. RCI can be continued during hospitalisation, except in cases of uncontrolled bacterial and fungal infection, sepsis or RCI attributed toxicity.

Panellists developed multiple consensus recommendations regarding management of adverse effects, including non-pharmacological interventions, pharmacological interventions and dose adjustments.

The Delphi method is a broadly accepted strategy for developing consensus recommendations based on expert opinion. The Delphi methodology dates back to the 1950s and was developed to create consensus in social science topics [28-34]. The methodology is particularly relevant when substantial experience and knowledge on a particular topic are available, but definitive experiments to prove the point are lacking. A key strength of the Delphi method is its use of a systematic, anonymous process that promotes free sharing of opinions and ideas, weighs all panellists' opinions equally, and makes it difficult for any individual panellist to dominate the process. E-mail was used to disseminate and collect the questionnaires; this methodology helped maintain the anonymous nature of the Delphi process.

The Delphi process has several limitations. There are no generally accepted criteria defining consensus in Delphi studies, and given the wide variety of topics investigated using the Delphi approach, it may not be possible to define generally applicable criteria $[32,33,35]$. Clearly the consensus represents a degree of agreement only among the experts and may be refuted in the future by rigorous studies $[30,31,35]$.

Panel selection and the development of the initial questionnaire may have inadvertently introduced bias into the process $[18,32]$. The panellists had a wide range of experience while satisfying the requirement for having prescribed RCI in treating patients with pulmonary sarcoidosis. These differing levels of expertise related to the study may have helped ensure that the panel reflected real-world practice and enabled the consensus recommendations to capture a full range of practice opinions [18]. Owing to lack of availability of RCI outside the USA, international panellists were not included. Other potential stakeholders, such as patients, pharmacists and insurance companies and other payers, were also not included.

Anonymity is an important aspect of the Delphi process, avoiding undue weight given to certain panellists. However, it also means that panellists are not accountable for their responses, possibly leading to responses based on insufficient or minimal consideration [35].

The companion articles in this issue of the European Respiratory Review, which concern phenotyping of sarcoidosis [36] and development of a treatment algorithm for sarcoidosis using the Delphi process [26], seem to suggest that the use of RCI, when considered, should be mainly for the advanced disease phenotype, where concomitant therapy with steroids and immunosuppressants has not successfully controlled the condition.

\section{Conclusion}

The modified Delphi process was successful in providing practical guidance on real-world issues in using RCI to treat patients with advanced sarcoidosis. There is a clear need for further studies to improve knowledge of dosing and titration to achieve the best results and discern the appropriate place for RCI in the portfolio of medications now used for advanced therapy.

Conflict of interest: F.F. Rahaghi reports grants and consulting fees from Mallinckrodt, during the conduct of the study. N.J. Sweiss has nothing to disclose. L.A. Saketkoo has nothing to declare. M.B. Scholand reports other from Boehringer Ingelheim, Genentech, Fibrogen and Global Blood Therapeutics, outside the submitted work. In addition, M.B. Scholand has a patent Apparatus, Compositions and Methods for Assessment of Chronic Obstructive Pulmonary Disease Progression among Rapid and Slow Decline Conditions issued. J.B. Barney has nothing to disclose. A.K. Gerke has nothing to disclose. E.E. Lower has nothing to disclose. M. Mirsaeidi reports grants and personal fees from Mallinckrodt, outside the submitted work. L. O'Hare has nothing to disclose. M.J. Rumbak has nothing to disclose. L. Samavati has participated in a Questcor Advisory Board Meeting in 2014 and received $\$ 6500$ compensation. R.P. Baughman reports grants and personal fees from Mallinckrodt, Novartis and Celgene, grants from Gilead, Genentech and Bayer, personal fees from West Pharmaceutical, during the conduct of the study.

Support statement: Editorial assistance was provided by AXON Communications (Edward K. Baldwin) and funded by an independent medical writing grant provided by Mallinckrodt Pharmaceuticals. Mallinckrodt played no role in the development, review, or approval to submit the manuscript for publication. These tasks were solely the responsibility of the authors. All authors had access to the Delphi questionnaire analysis and data and participated in the review, revision and approval of the content of the manuscript for submission.

\section{References}

1 Hunninghake GW, Costabel U, Ando M, et al. ATS/ERS/WASOG statement on sarcoidosis. American Thoracic Society/European Respiratory Society/World Association of Sarcoidosis and other Granulomatous Disorders. Sarcoidosis Vasc Diffuse Lung Dis 1999; 16: 149-173. 
2 Baughman RP, Drent M, Kavuru M, et al. Infliximab therapy in patients with chronic sarcoidosis and pulmonary involvement. Am J Respir Crit Care Med 2006; 174: 795-802.

3 Casanova N, Zhou T, Knox KS, et al. Identifying novel biomarkers in sarcoidosis using genome-based approaches. Clin Chest Med 2015; 36: 621-630.

$4 \quad$ Iannuzzi MC, Rybicki BA, Teirstein AS. Sarcoidosis. N Engl J Med 2007; 357: 2153-2165.

5 Baughman RP. Sarcoidosis. Clin Dermatol 2007; 25: 231.

6 Judson MA. The clinical features of sarcoidosis: a comprehensive review. Clin Rev Allergy Immunol 2015; 49: 63-78.

7 Swigris JJ, Olson AL, Huie TJ, et al. Sarcoidosis-related mortality in the United States from 1988 to 2007. Am J Respir Crit Care Med 2011; 183: 1524-1530.

8 Mirsaeidi M, Machado RF, Schraufnagel D, et al. Racial difference in sarcoidosis mortality in the United States. Chest 2015; 147: 438-449.

9 Kouranos V, Jacob J, Wells AU. Severe sarcoidosis. Clin Chest Med 2015; 36: 715-726.

10 Soto-Gomez N, Peters JI, Nambiar AM. Diagnosis and management of sarcoidosis. Am Fam Physician 2016; 93: 840-848.

11 Al-Kofahi K, Korsten P, Ascoli C, et al. Management of extrapulmonary sarcoidosis: challenges and solutions. Ther Clin Risk Manag 2016; 12: 1623-1634.

12 Baughman RP, Grutters JC. New treatment strategies for pulmonary sarcoidosis: antimetabolites, biological drugs, and other treatment approaches. Lancet Respir Med 2015; 3: 813-822.

13 James WE, Baughman R. Treatment of sarcoidosis: grading the evidence. Expert Rev Clin Pharmacol 2018; 11: 677-687.

14 Miller MA, Bass HE. Effect of Acthar-c (ACTH) in sarcoidosis. Ann Intern Med 1952; 37: 776-784.

15 Salomon A, Appel B, Collins SF, et al. Sarcoidosis: pulmonary and skin studies before and after ACTH and cortisone therapy. Dis Chest 1956; 29: 277-291.

16 Baughman RP, Barney JB, O'Hare L, et al. A retrospective pilot study examining the use of Acthar gel in sarcoidosis patients. Respir Med 2016; 110: 66-72.

17 Baughman RP, Sweiss N, Keijsers R, et al. Repository corticotropin for chronic pulmonary sarcoidosis. Lung 2017; 195: 313-322.

18 Hsu CC, Sandford BA. The Delphi technique: making sense of consensus. Practical Assessment, Research \& Evaluation 2007; 12: 1-8. Available from: https://scholarworks.umass.edu/pare/vol12/iss1/10

19 Delbecq AL, van de Ven AH, Gustafson DH. Group techniques for program planning. Glenview, Foresman, and Co, 1975.

20 Hasson F, Keeney S, McKenna H. Research guidelines for the Delphi survey technique. J Adv Nurs 2000; 32: $1008-1015$

21 de Meyrick J. The Delphi method and health research. Health Education 2003; 103: 7-16.

22 Rahaghi FF, Feldman JP, Allen RP, et al. Recommendations for the use of oral treprostinil in clinical practice: a Delphi consensus project. Pulm Circ 2017; 7: 167-174.

23 Rahaghi FF, Alnuaimat HM, Awdish RLA, et al. Recommendations for the clinical management of patients receiving macitentan for pulmonary arterial hypertension (PAH): a Delphi consensus document. Pulm Circ 2017; 7: 702-711.

24 Chopra I, Qin Y, Kranyak J, et al. Repository corticotropin injection in patients with advanced symptomatic sarcoidosis: retrospective analysis of medical records. Ther Adv Respir Dis 2019; 13: 1753466619888127.

25 Philbin M, Niewoehner J, Wan GJ. Clinical and economic evaluation of repository corticotropin injection: a narrative literature review of treatment efficacy and healthcare resource utilization for seven key indications. $A d v$ Ther 2017; 34: 1775-1790.

26 Rahaghi FF, Baughman RP, Saketkoo LA, et al. Delphi consensus recommendations for a treatment algorithm in pulmonary sarcoidosis. Eur Respir Rev 2020; 29: 190146.

27 Lower EE, Sturdivant M, Grate L, et al. Use of third-line therapies in advanced sarcoidosis. Clin Exp Rheumatol 2019; in press.

28 Schutt AC, Bullington WM, Judson MA. Pharmacotherapy for pulmonary sarcoidosis: a Delphi consensus study. Respir Med 2010; 104: 717-723.

29 Haines S, Baker T, Donaldson M. Development of a physical performance assessment checklist for athletes who sustained a lower extremity injury in preparation for return to sport: a Delphi study. Int J Sports Phys Ther 2013; 8: 44-53

30 Li Y, Ehiri J, Hu D, et al. Framework of behavioral indicators for outcome evaluation of TB health promotion: a Delphi study of TB suspects and TB patients. BMC Infect Dis 2014; 14: 268.

31 Mansell G, Shapley M, van der Windt D, et al. Critical items for assessing risk of lung and colorectal cancer in primary care: a Delphi study. Br J Gen Pract 2014; 64: e509-e515.

32 Phillips AC, Lewis LK, McEvoy MP, et al. Protocol for development of the guideline for reporting evidence based practice educational interventions and teaching (GREET) statement. BMC Med Educ 2013; 13: 9.

33 Phillips AC, Lewis LK, McEvoy MP, et al. A Delphi survey to determine how educational interventions for evidence-based practice should be reported: stage 2 of the development of a reporting guideline. BMC Med Educ 2014; 14: 159.

34 Pietersma S, de Vries M, van den Akker-van Marle ME. Domains of quality of life: results of a three-stage Delphi consensus procedure among patients, family of patients, clinicians, scientists and the general public. Qual Life Res 2014; 23: 1543-1556.

35 Powell C. The Delphi technique: myths and realities. J Adv Nurs 2003; 41: 376-382.

36 Baughman RP, Scholand MB, Rahaghi FF. Clinical phenotyping: role in treatment decisions in sarcoidosis. Eur Respir Rev 2020; 29: 190145. 